\title{
Persistent environmental pollutants and couple fecundity: an overview
}

\author{
Germaine M Buck Louis \\ Division of Intramural Population Health Research, Eunice Kennedy Shriver National Institute of Child Health \\ and Human Development, National Institutes of Health, 6100 Executive Blvd., Room 7B03, Rockville, \\ Maryland 20852, USA \\ Correspondence should be addressed to G M Buck Louis; Email: louisg@mail.nih.gov
}

\begin{abstract}
Speculation has arisen that human fecundity may be declining, possibly a function of exposure to persistent environmental chemicals that resist degradation resulting in various pathways for human exposure. In contrast to considerable animal evidence suggesting adverse effects of such chemicals on reproduction, limited human research has been undertaken. To date, available data stem largely from ten unique study cohorts that have quantified individual chemical exposures in relation to time-to-pregnancy (TTP), which is a measure of couple fecundity. Diminished fecundability odds ratios indicative of longer TTP were observed in all but two studies, although not all findings achieved statistical significance. Persistent chemicals associated with reduced couple fecundity as measured by a longer TTP included $\beta \mathrm{HCH}$, cadmium, lead, mercury, 1,1-dichloro-2,2-bis(p-chlorophenyl)ethylene, TCCD dioxin, and select polybrominated diethers, polychlorinated biphenyls, and perfluorochemicals. Important methodologic limitations need to be considered in weighing the evidence: i) reliance on pregnant women, which may exclude women with the highest exposures if related to the inability to conceive; ii) retrospectively reported TTP, which may be associated with bidirectional reporting errors; and iii) limited attention to male partners or couples' exposures. While current evidence is not inconsistent with animal evidence, concerted efforts to address lingering data gaps should include novel strategies for recruiting couples, the longitudinal measurement of TTP, and the continued enrollment of couples across successive pregnancies. This latter strategy will provide a more complete understanding of the toxicokinetics of chemicals during sensitive windows and their implications for fecundity and its related impairments.
\end{abstract}

Reproduction (2014) 147 R97-R104

\section{Introduction}

An evolving body of evidence suggests that human fecundity, defined as the biologic capacity of men and women for reproduction irrespective of pregnancy intentions (Buck Louis 2011), may be declining raising concerns about the sustainability of some populations (Daguet 2002, Lutz et al. 2003, Skakkebaek et al. 2006).

This paper forms part of a special issue of Reproduction on Endocrine Disrupters. This article was presented at the 7 th Copenhagen Workshop on Endocrine Disrupters, 28-31 May 2013. The meeting was supported by the Danish Ministry of the Environment - Environmental Protection Agency as an activity under the Danish Centre on Endocrine Disrupters. Publication of this special issue has been supported by the Society for Reproduction and Fertility. The opinions or views expressed in this special issue are those of the authors, and do not necessarily reflect the opinions or recommendations of the Danish Ministry of the Environment - Environmental Protection Agency or the Society for Reproduction and Fertility. The Guest Editors for this special issue were Anna-Maria Andersson, Hanne Frederiksen, Niels Erik Skakkebæk, Rigshospitalet, Denmark, Kenneth M Grigor, Western General Hospital, Edinburgh, UK and Jorma Toppari, University of Turku, Finland.
While controversial in many regards, evidence consistent with diminishing male fecundity includes declining semen quality reported by some authors (Zou et al. 2011, Geoffroy-Siraudin et al. 2012) but not all as recently summarized (Fisch \& Braun 2013), along with higher genital-urinary malformation rates among men with fecundity impairments or reproductive site cancers in comparison to unaffected individuals (Bray et al. 2006, Skakkebaek et al. 2006, 2007, Saravelos et al. 2008). The relatively high prevalence rates of fibroids, polycystic ovarian syndrome, and endometriosis (Baird et al. 2003, Azziz et al. 2004, Gylfason et al. 2010) may be suggestive of diminishing female fecundity, while increasing infertility rates may be indicative of diminished couple fecundity (Priskorn et al. 2012, Thoma et al. 2013).

Fecundity is now recognized to have implications across the lifespan. For example, boys born with genitalurinary malformations are at increased risk of alterations in semen quality, infertility, and testes cancer in adulthood than unaffected boys (Trussell \& Lee 2004, Bray et al. 2006). In fact, recent authors have reported semen quality to be positively associated with longevity 
(Jensen et al. 2009). Associations between female fecundity and later onset disease have also been reported. For example, girls born small-for-gestation are reported to have poorer adult ovarian development and function relative to adequately sized girls (Ibáñez et al 2000). Girls with low birth weights irrespective of gestation were reported to have biochemical and clinical features characteristic of polycystic ovarian syndrome (Pandolfi et al. 2008). Similarly, women with polycystic ovaries are at increased risk of gravid disease and metabolic disorders later in adulthood (Talbott et al. 2004). Infertility was also observed to be associated with gravid diseases such as gestational diabetes (Tobias et al. 2013). Collectively, the findings in males and, subsequently, females have been conceptualized as suggesting an early origin for onset, or the so-called testicular and ovarian dysgenesis syndromes (Skakkebaek et al. 2001, Buck Louis et al. 2011a).

Potential reasons for declining fecundity are largely unexplored, although environmental factors are suggested and serve as the impetus for this paper. Attention is directed to persistent chemicals that resist degradation, as indicative by their long half-lives spanning several years for some compounds (www. cdc.gov/exposurereport/pdf/FourthReport.pdf). Such chemicals include 1,1-dichloro-2,2-bis(p-chlorophenyI)ethylene (DDE) and its parent compound 1,1,1trichloro-2,2-bis(p-chlorophenyl)ethane (DDT), dioxin, metals, organochlorine pesticides (OCPs), polybrominated biphenyls (PBBs), polybrominated diethers (PBDEs), polychlorinated biphenyls (PCBs), and perfluorochemicals (PFCs). This paper is organized as three questions and concludes with a summary of chemicals reported to adversely affect fecundity using a weight of evidence approach.

\section{How can couple fecundity be assessed relative to environmental chemicals?}

A range of possible outcomes can be used to assess either male or female fecundity when considered individually. By contrast, couple fecundity is largely measured by time-to-pregnancy (TTP), which is defined as the number of menstrual cycles or calendar months required to become pregnant. TTP is used globally as a marker of how quickly a couple becomes pregnant, or not. It can also be categorized to denote fecundity-related impairments such as conception delay (TTP, greater than six cycles per month) or infertility (>12 cycles/month) recognizing that neither of these two impairments denotes sterility without further medical investigation.

In prospectively followed couples attempting pregnancy, $\sim 80 \%$ of women achieve pregnancy within six cycles of trying (Bonde et al. 1998, Buck Louis et al. $2011 b$ ), while $13-18 \%$ of couples do not achieve pregnancy within 12 months (Zinman et al. 1996,
Buck Louis et al. 2012). Of note are the regional differences in TTP (Juul et al. 1999, Sanin et al. 2009). The extent to which such differences in TTP reflect regional variations in semen quality (Jørgensen et al. 2001, Punab et al. 2002, Swan et al. 2003) remains to be established.

One important limitation of using TTP as a measure of couple fecundity is that it provides no information as to whether the delays are male, female, or couple mediated. Much of the available research relies on females, not couples. A second data gap is the absence of research that has empirically assessed how male (e.g. semen quality) and female (e.g. ovulatory cycles) fecundity jointly mediates couple fecundity or TTP. This data gap likely reflects the few prospective cohort studies with preconception enrollment of couples conducted to date (Buck et al. 2004).

While TTP can be estimated with the use of prospective and retrospective designs, the former is considered the gold standard given its ability to longitudinally measure at risk time and incident pregnancy along with other time-varying lifestyle factors such as alcohol consumption or smoking. While reliability is reported to be good for retrospectively measured TTP even after long period of recall (Joffe et al. 1993), its validity is only good for short recall or within 3-20 months (Zieluis et al. 1992). However, it has poor validity for longer periods of recall as reflected in bidirectional errors or the underand over-reporting of TTP by women (Cooney et al. 2009). Another important methodologic limitation underlying the use of retrospective TTP is digit preference reporting (Ridout \& Morgan 1991).

The fecundability odds ratio (FOR) estimates the probability of pregnancy each menstrual cycle or month, given exposure and conditional on not having achieved pregnancy in the previous cycle. FORs are estimated along with their $95 \% \mathrm{Cl}$ for assessing significance. A FOR $<1.0$ denotes reduced fecundability or a longer TTP, whereas an FOR $>1.0$ denotes enhanced fecundability or a shorter TTP. Despite increasing recognition of the importance of lifestyle factors for TTP (Rothman et al. 2013), only $14 \%$ of the variation in TTP was explained by oral contraceptive use, cycle length, age, and parity at the population level, whereas other lifestyle factors were not retained in models (Axmon et al. 2006a). This finding underscores our limited understanding of the population and individual level determinants of human fecundity and is an important consideration when assessing environmental chemicals.

\section{What research has focused on persistent chemicals and couple fecundity?}

\section{Research relying on retrospectively collected TTP}

Very little research has focused on persistent environmental chemicals and couple fecundity, despite many 
such compounds having been quantified in semen, follicular, and genital track fluid (Wagner et al. 1990, DeFelip et al. 2004, Jirsová et al. 2010). To date, much available research relies on retrospectively ascertained TTP from pregnant women or women with unique residential or lifestyle (i.e. fish consumption) exposures. Axmon et al. (2004) queried 183 sisters of fishermen about TTP and obtained a blood sample for the quantification of plasma PCB congener $153 \sim 20$ years following the first planned pregnancy necessitating the need to backwardly extrapolate exposure at the relevant time period for TTP. A positive association was observed suggesting enhanced fecundity or a shorter TTP, including for another subset of wives of fisherman. The findings, however, did not achieve significance. In a subsequent study, Axmon et al. (2006b) recruited pregnant women and their male spouses from Greenland, Kharkiv, and Warsaw and queried them about TTP. FORs $<1.0$ were observed for male and female serum PCB 153 in Greenland and Kharkiv but not in Warsaw, and female DDE concentrations were also associated with FORs $<1.0$ but only in Greenland. However, only the findings for female exposures in Greenland achieved significance. Gesink Law et al. (2005) utilized the historic U.S. Collaborative Perinatal Project that enrolled pregnant women from 12 clinical sites in the USA, 1959-1965. Banked serum was analyzed for 390 women for the quantification of 11 PCBs, DDT, and DDE. Women in the highest quintile of PCBs and DDE had $\mathrm{a} \approx 35 \%$ reduction in fecundity or a longer TTP when compared to women in the lowest categories. However, the findings failed to reach significance.

Harley et al. (2008) assessed serum DDT and DDE concentrations in 289 pregnant migrant farmworkers participating in the CHAMACOS cohort study in relation to retrospectively collected TTP. FORs were all below one, reflecting reduced (2-9\%) fecundity for $o, p^{\prime}$-DDT, $p, p^{\prime}$-DDT, and $p, p^{\prime}$-DDE respectively. Subsequently, Harley et al. (2010) assessed ten serum PBDE congeners for a subset of pregnant women in the CHAMACOS cohort. Only PBDE congener 100 was associated with a significant $40 \%$ reduction in fecundity, with findings robust to additional sensitivity analyses given their reliance on retrospective TTP.

Cole et al. (2005) utilized a cross-sectional design to quantify OCPs, PCBs, and metals in 41 first-time pregnant couples. Only female blood mercury was associated with reduced fecundity, conferring a $78 \%$ significant reduction in fecundity. Dioxin and TTP has been assessed in one study. Specifically, Eskenazi et al. (2010) assessed serum 2,3,7,8-tetrachlorodibenzop-dioxin concentrations in 278 women that were extrapolated back to the time women were attempting pregnancy following a dioxin plant explosion in relation to retrospectively reported TTP for 278 (28\%) women. Fecundity was reduced $\approx 25 \%$, and a twofold higher odds of infertility or achieving pregnancy after $12+$ months of trying was also observed for participants.

With regard to PFCs, Fei et al. (2009) utilized banked biospecimens for the quantification of plasma perfluorooctanoate (PFOA) and perfluorooctane sulfonate (PFOS) concentrations among a subset of 1240 pregnant women who participated in the Danish National Birth Cohort and who were queried about TTP during pregnancy. Significant inverse trends were observed for both PFOA and PFOS and TTP, reflecting an $\sim 30 \%$ reduction in fecundity for women in the highest three quartiles relative to women in the lowest.

Two other pregnancy studies are worth mentioning despite not being directly comparable to research with individual chemical concentrations and TTP. Whitworth et al. (2012) assessed plasma PFOA and PFOS concentrations in 910 pregnant women participating in the Norwegian Mother and Child (MoBA) cohort study with retrospectively collected TTP dichotomized as requiring $>12$ months for pregnancy vs $\leq 12$ months. Parous but not nulliparous women in the highest quartiles of PFOA and PFOS had a significant twofold higher odds of a TTP $>12$ months in comparison to women with lower concentrations. In the PELAGIE cohort, pregnant mothers were queried about their TTP in relation to 14 OCPs, 12 PCBs, and ten PBDEs that were quantified in the cord blood of 394 infants (Chevrier et al. 2013). Negative associations were observed for most compounds, particularly for total PCBs (54\% reduction), $p, p^{\prime}$-DDE (40\% reduction), and two OCPs $(\beta \mathrm{HCH}$ and $\mathrm{HCB}, 39$ and $10 \%$ respectively).

\section{Research with prospectively measured TTP}

Two prospective cohort studies with preconception enrollment of women have assessed PCBs and PFCs. Buck Louis et al. (2009) recruited 83 women upon discontinuing contraception with daily follow-up through 12 menstrual cycles at risk for pregnancy in the New York State Angler Cohort Study (NYSACS). Both estrogenic and anti-estrogenic PCBs were associated with $\approx 68 \%$ reduction or more in fecundity, although the findings did not achieve significance. A second prospective cohort study with preconception recruitment of couples followed for 6 months reported by Vestergaard et al. (2012) utilized banked serum from 222 women who participated in the Danish First-Pregnancy Planners Cohort, 1992-1995 (Bonde et al. 1998). Of the eight PFCs considered, two metabolites - EtFOSAA and MeFOSS - conferred FORs $<1.0$ reflecting a 20 and $10 \%$ reduction respectively. However, the findings did not achieve significance.

The most recently conducted prospective cohort study with preconception enrollment of both partners of the couple for the specific investigation of environmental influences on human fecundity is the Longitudinal Investigation of Fertility and the Environment (LIFE) 
Study (Buck Louis et al. 2011b). Couples were recruited from targeted geographic areas with reported exposures to persistent compounds upon discontinuing contraception. TTP was longitudinally measured using a combination of data from the daily journals completed by both partners and the Clearblue Fertility Monitor, which provided visual prompts to help couples time intercourse relative to ovulation. Pregnancy denoted a positive home pregnancy test on the day of expected menstruation using digital home test kits. Various environmental compounds were associated with diminished fecundity, and surprising few were associated with enhanced fecundity as measured by FORS $>1$. Specifically, female blood cadmium and male lead concentrations were associated with a 22 and $15 \%$ reduction in fecundity respectively when modeled individually (Buck Louis et al. 2012). When both partners' blood metals were jointly modeled given the low correlation between partners, male lead concentration continued to reflect an $18 \%$ reduction in fecundity. All findings remained significant even after adjusting for relevant covariates. Also, female serum concentrations of PCB congeners 118, 167, and 209, and perfluorooctane sulfonamide (PFOSA) were consistently associated with diminished fecundity, ranging from 18 to $21 \%$ (Buck Louis et al. 2013). Among male partners, $p, p^{\prime}-\mathrm{DDE}$ and PCB congeners 138, 156, 157, 167, 170, 172, and 209 were significantly associated with reduced (17-29\%) fecundity denoting a longer TTP. Male partners' concentration of PCB 101 was the only chemical significantly associated with enhanced fecundity or a shorter TTP.

Table 1 summarizes the weight of evidence reported by ten different study cohorts that had individual chemical measurements and data on either retrospective or prospective TTP data. This summary table reflects the sparse available data and a preponderance of data relying on pregnant women, retrospective TTP, and the limited attention to male partners. Despite these challenges, all but two studies reported FORs $<1.0$ for at least one chemical suggesting an association with diminished couple fecundity. However, not all the findings achieved significance. Findings from the LIFE Study corroborate earlier reports, including for female PCB concentrations (Axmon et al. 2006b), p, p'-DDE (Harley et al. 2008) when based on male concentrations, and PFOS and PFOSA (Fei et al. 2009). Of note is the observation that the magnitude of FORs reported for various persistent chemicals is relatively smaller than those reported for biologic determinants such as oligospermia or gynecologic disorders (i.e. FORs 0.34 and 0.46 respectively) (Vestergaard et al. 2012), but comparable for those reported for cigarette smoking or serum cotinine concentrations, higher BMls, and parental ages (Buck Louis et al. 2012, Chevrier et al. 2013).

\section{What are the next steps for answering data gaps?}

Globally, two avenues of research may offer insight regarding the relationship between environmental chemicals and couple fecundity. One avenue is to continue to leverage existing pregnancy or birth cohort studies. A number of recent pregnancy cohort studies have been implemented in the past decade, and most have banked biospecimens that may be suitable for continued investigation. Still, such effort will be limited by reliance on women successfully achieving pregnancy and retrospective TTP. If exposures prevent couples from achieving pregnancy, they will be excluded from the study cohort and possibly impact study conclusions. Still, it may be possible to devise strategies to measure exposures of women not achieving pregnancy to empirically assess this lingering question and to foster data-driven decision-making. A second promising avenue is to leverage children from existing birth cohorts and to design prospective TTP studies when they enter reproductive years. This approach will provide information on the woman's in utero exposure and also her exposure at the time she is interested in becoming pregnant. The same would be true for males. Ideally, it would be important to follow couples through all their pregnancy-trying attempts to obtain data relevant for understanding the toxicokinetics of chemicals and their impact on sensitive fecundity endpoints across successive pregnancy attempts. Such an approach would be highly informative for the proper modeling of exposures and reproductive outcomes in the context of a couple's past reproductive performance (e.g. parity) and other relevant factors such as age. Irrespective of approach or any others that may be relevant, every effort should be made to quantify exposures in both partners in keeping with the couple dependent nature of human reproduction. Failure to consider male partners may result in erroneous conclusions when based solely on female exposures.

With increased recognition of the need to model chemical mixtures in keeping with the nature of human exposure, continual efforts to develop statistical models for handling correlated and hierarchical data characteristic of couple-based designs are urgently needed. This work becomes more challenging when attempting to include lifestyle and diet to identify potential modifying factors that may minimize the effects stemming from internal chemical doses and, thereby, promote health and well being. While beyond the focus of this paper, future work should also include measurement of both persistent and non-persistent chemicals, given the growing evidence suggesting an adverse relationship between short-lived compounds such as bisphenol $A$ and phthalates and a spectrum of reproductive endpoints. Adverse effects reported include alterations in hormonal milieu, reduced number of oocytes retrieved, and implanted among couples undergoing assisted reproductive technologies and alterations 


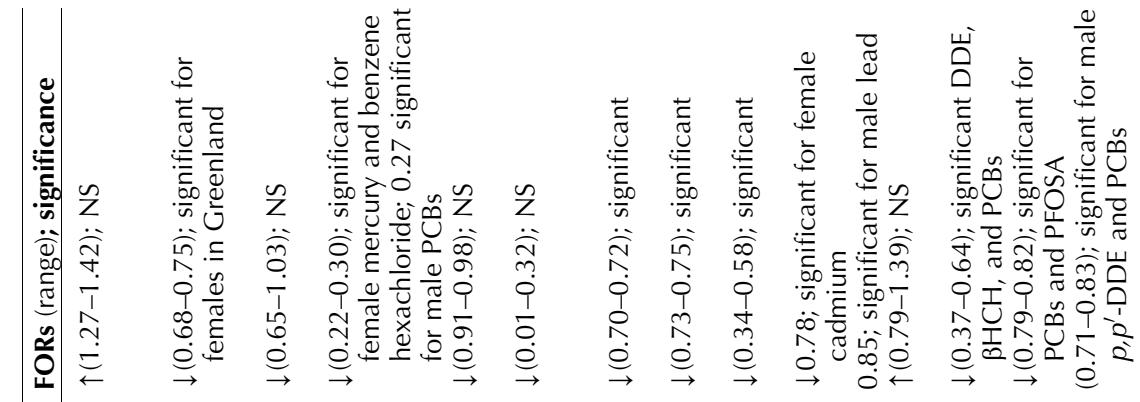

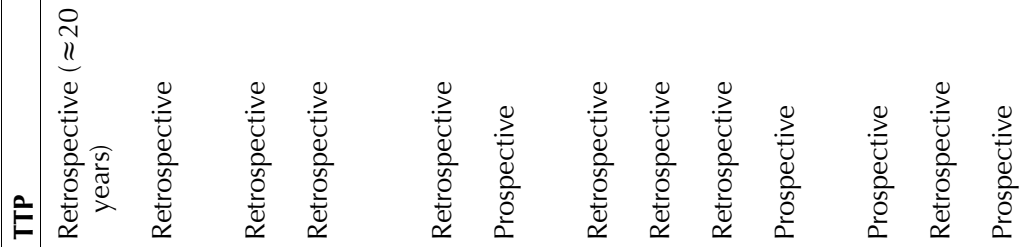

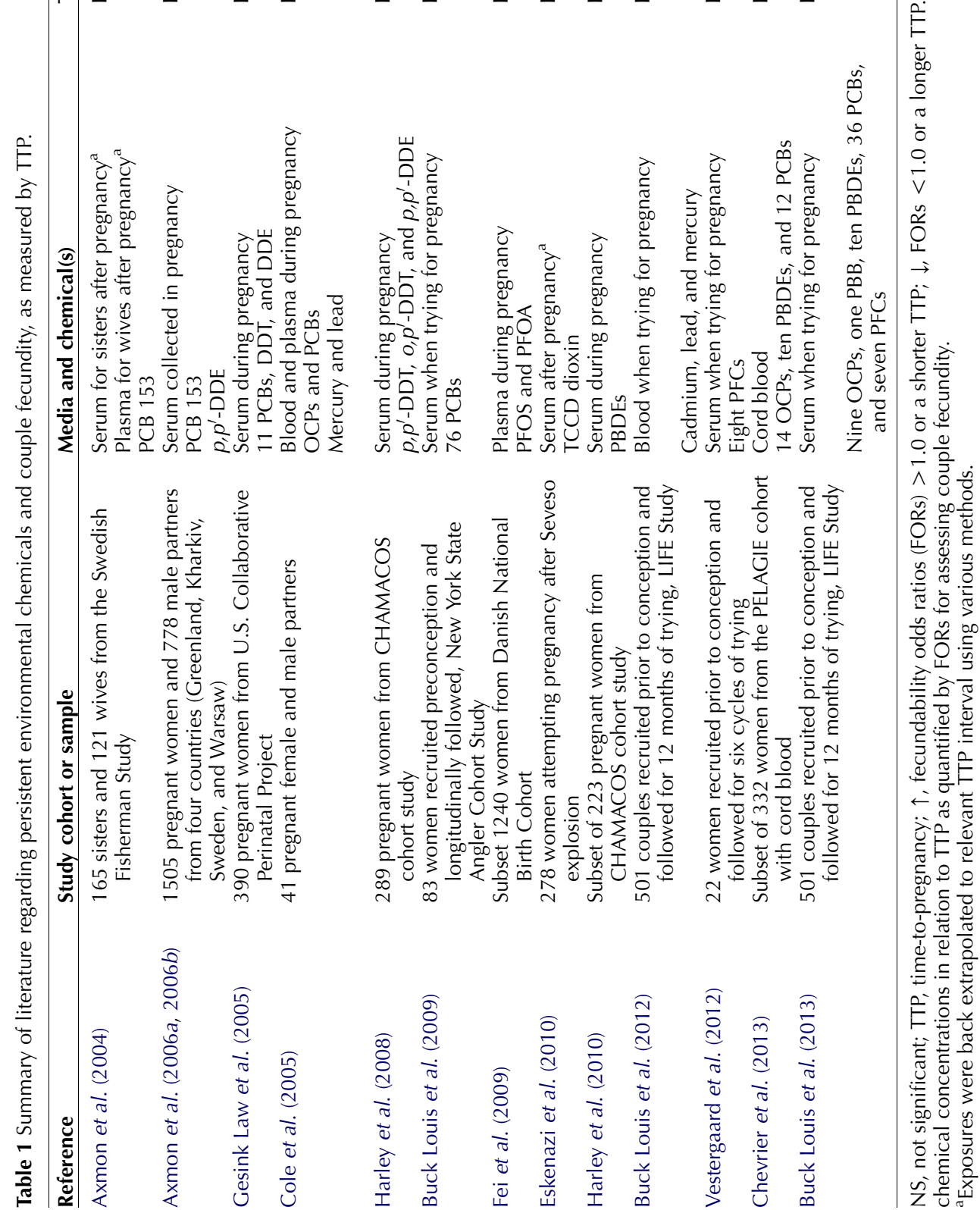


in semen quality (Duty et al. 2003, Jönsson et al. 2005, Mendiola et al. 2010, Mok-Lin et al. 2010, Ehrlich et al. 2012). Perhaps, efforts such as Environmental Wide Association Studies (EWAS) may be one approach for considering all environmental chemicals, but others options are likely to emerge in the near future. Continued efforts are also needed to resolve lingering laboratory analytical issues, such as the ideal modeling of chemical concentrations below the laboratory limits of detection or alternatives to the automatic adjustment of chemicals for serum lipids or urinary creatinine when assessing potential reproductive toxicity.

\section{Conclusion}

An evolving body of observational research suggests that environmentally relevant concentrations of select persistent environmental chemicals may be affecting human fecundity, as evident as a longer time required for achieving pregnancy. Such subtle changes may easily be missed without continued and purposeful research aimed at the preconception enrollment of couples for longitudinal measurement of sensitive outcomes such as TTP and pregnancy loss. Male-mediated exposures also are important and failure to consider them when assessing couple-dependent outcomes such as TTP or pregnancy loss may result in erroneous conclusions, particularly in the absence of female exposures. Future research will require sophisticated analytic methods that are well grounded within human biology and capable of handling the hierarchical and correlated structure of chemical exposures as we seek to delineate and quantify threats to human fecundity. In the context of emerging chemical signals potentially relevant for human fecundity, this author urges shared collaboration and creative utilization of existing resources from which to answer lingering data gaps. The excellent work reported above that utilized banked biospecimens from pregnancy cohort studies is a step in the right direction but cannot replace the need for prospective cohort studies with preconception enrollment of couples. Novel strategies aimed at recruiting contemporary birth cohorts who are or will be soon testing their fecundity are needed. Such empirical evidence is needed for informing public policy and informed decision-making.

\section{Declaration of interest}

The author declares that there is no conflict of interest that could be perceived as prejudicing the impartiality of the review reported.

This article is based on work presented at the 7 th Copenhagen Workshop on Endocrine Disrupters, which was supported by the Danish Ministry of the Environment Environmental Protection Agency. Publication of this special issue was supported by the Society for
Reproduction and Fertility. The author declares no known or possible conflicts with the sponsors of the COW meeting.

\section{Funding}

This study is supported by the Intramural Research Program of the Eunice Kennedy Shriver National Institute of Child Health and Human Development. Funding for the LIFE Study was provided by contracts N01-HD-3-3355; N01-HD-3-3356; and $\mathrm{NOH}-\mathrm{HD}-3-3358$.

\section{References}

Axmon A, Rylander L, Strömberg U, Jönsson B, Nilsson-Ehle P \& Hagmar L 2004 Polychlorinated biphenyls in serum and time to pregnancy. Environmental Research 96 186-195. (doi:10.1016/j.envres.2003. 10.002)

Axmon A, Rylander L, Albin M \& Hagmar L 2006a Factors affecting time to pregnancy. Human Reproduction 21 1279-1284. (doi:10.1093/humrep/ dei469)

Axmon A, Thulstrup A-M, Rignell-Hybom A, Pedersen HS, Zvyezday V, Ludwicki JK, Jönsson BA, Toft G, Bonde J-P, Hagmar L et al. 2006 b Time to pregnancy as a function of male and female serum concentrations

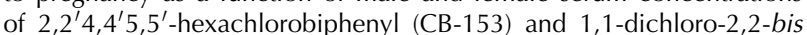
( $p$-chlorophenyl)-ethylene ( $p^{\prime} p$-DDE). Human Reproduction 21 657-665. (doi:10.1093/humrep/dei397)

Azziz R, Woods KS, Reyna R, Key TJ, Knochenhauer ES \& Yildiz BO 2004 The prevalence and features of the polycystic ovary syndrome in an unselected population. Journal of Clinical Endocrinology and Metabolism 89 2745-2749. (doi:10.1210/jc.2003-032046)

Baird DD, Dunson DB, Hill MC, Cousins D \& Schectman JM 2003 High cumulative incidence of uterine leiomyoma in black and white women: ultrasound evidence. American Journal of Obstetrics and Gynecology 188 100-107. (doi:10.1067/mob.2003.99)

Bonde JP, Hjollund NH, Jensen TK, Ernst E, Kolstad H, Henriksen TB, Giwercman A, Skakkebaek NE, Andersson AM \& Olsen J 1998 A followup study of environmental and biologic determinants of fertility among 430 Danish first-pregnancy planners: design and methods. Reproductive Toxicology 12 19-27. (doi:10.1016/S0890-6238(97)00096-8)

Bray F, Ferlay J, Devessa SS, McGlynn KA \& Møller H 2006 Interpreting the international trends in testicular seminoma and nonseminoma incidence. Nature Clinical Practice. Urology 3 532-543. (doi:10.1038/ ncpuro0606)

Buck GM, Lynch CD, Stanford JB, Sweeney AM, Schieve LA, Rockett JC, Selevan SG \& Schrader SM 2004 Prospective pregnancy study designs for assessing reproductive developmental toxicants. Environmental Health Perspectives 112 79-86. (doi:10.1289/ehp.6262)

Buck Louis GM 2011 Fecundity and fertility. In Reproductive and Perinatal Epidemiology, pp 16-61. Eds GM Buck Louis \& RW Platt. New York: Oxford University Press.

Buck Louis GM, Dmochowsk J, Lynch CD, Kostyniak PJ, McGuinness BM \& Vena JE 2009 Polychlorinated biphenyl concentrations, lifestyle and time-to-pregnancy. Human Reproduction 24 451-458. (doi:10.1093/ humrep/den373)

Buck Louis GM, Cooney MA \& Peterson CM 2011a The ovarian dysgenesis syndrome. Journal of Developmental Origins of Health and Disease 2 25-35. (doi:10.1017/S2040174410000693)

Buck Louis GM, Schisterman EF, Sweeney AM, Wilcosky TC, GoreLangton R, Lynch CD, Barr DD, Schrader SM, Kim S, Chen Z et al. 2011 b Designing prospective cohort studies for assessing reproductive and developmental toxicity during sensitive windows of human reproduction and development - the LIFE Study. Paediatric and Perinatal Epidemiology 25 413-424. (doi:10.1111/j.1365-3016.2011.01205.x)

Buck Louis GM, Sundaram R, Schisterman EF, Sweeney AM, Lynch CD, Gore-Langton RE, Chen Z, Kim S, Caldwell K \& Boyd Barr D 2012 Heavy metals and couple fecundity, the LIFE Study. Chemosphere $\mathbf{8 7}$ 1201-1207. (doi:10.1016/j.chemosphere.2012.01.017) 
Buck Louis GM, Sundaram R, Schisterman EF, Sweeney AM, Lynch CD, Gore-Langton RE, Maisog J, Kim S, Chen Z \& Barr DB 2013 Persistent environmental pollutants and couple fecundity, the LIFE Study. Environmental Health Perspectives 121 231-236. Epub 2012 Oct 31. (doi:10.1289/ehp.1205301)

Chevrier C, Warembourg C, Gaudreau E, Monfort C, Le Blanc A, Guldner L \& Cordier S 2013 Organochlorine pesticides, polychlorinated biphenyls, seafood consumption, and time-to-pregnancy. Epidemiology 24 1-10. (doi:10.1097/EDE.0b013e31827f53ec)

Cole DC, Wainman B, Sanin LH, Weber F-P, Muggah H \& Ibrahim S 2005 Environmental contaminant levels and fecundability among nonsmoking couples. Reproductive Toxicology 22 13-19. (doi:10.1016/ j.reprotox.2005.12.001)

Cooney MA, Buck Louis G, Sundaram R, McGuiness BM \& Lynch CD 2009 Validity of self-reported time to pregnancy. Epidemiology 26 56-59. (doi:10.1097/EDE.0b013e31818ef47e)

Daguet F 2002 Un siecle de fecondite francaise: 1901-1999. INSEE, Paris.

DeFelip E, di Domenico A, Miniero R \& Silvestroni L 2004 Polychlorobiphenyls and other organochlorine compounds in human follicular fluid. Chemosphere 54 1445-1449. (doi:10.1016/j.chemosphere.2003. 10.040)

Duty SM, Silva MJ, Barr DB, Brock JW, Ryan L, Chen Z, Herrick RF, Christiani DC \& Hauser R 2003 Phthalate exposure and human semen parameters. Epidemiology 14 269-277. (doi:10.1097/01.EDE.00000 59950.11836.16)

Ehrlich S, Williams PL, Missmer SA, Flaws JA, Berry KF, Calafat AM, Ye X, Petrozza JC, Wright D \& Hauser R 2012 Urinary bisphenol A concentrations and implantation failure among women undergoing in vitro fertilization. Environmental Health Perspectives 120 978-983. (doi:10.1289/ehp.1104307)

Eskenazi B, Warner M, Marks AR, Samuels S, Needham L, Brambilla P \& Mocarelli P 2010 Serum dioxin concentrations and time to pregnancy. Epidemiology 21 224-231. (doi:10.1097/EDE.0b013e 3181cb8b95)

Fei C, McLaughlin JK, Lipworth L \& Olsen J 2009 Maternal levels of perfluorinated chemicals and subfecundity. Human Reproduction $\mathbf{2 4}$ 1200-1205. (doi:10.1093/humrep/den490)

Fisch H \& Braun SR 2013 Trends in global semen parameter values. Asian Journal of Andrology 15 169-173. (doi:10.1038/aja.2012.143)

Geoffroy-Siraudin C, Loundou AD, Romain F, Achard V, Courbière B, Perrard MH, Durand P \& Guichaoua MR 2012 Decline of semen quality among 10932 males consulting for couple infertility over a 20-year period in Marseille, France. Asian Journal of Andrology 14 584-590. (doi:10.1038/aja.2011.173)

Gesink Law DC, Klebanoff MA, Brock JW, Dunson DB \& Longnecker MP 2005 Maternal serum levels of polychlorinated biphenyls and 1,1-dichloro-2,2-bis(p-chlorophenyl)ethylene (DDE) and time to pregnancy. American Journal of Epidemiology 162 523-532. (doi:10.1093/ aje/kwi240)

Gylfason JT, Kristjansson KA, Sverrisdottir G, Jonsdottir K, Rafnsson V \& Geirsson RT 2010 Pelvic endometriosis diagnosed in an entire nation over 20 years. American Journal of Epidemiology 172 237-243. (doi:10.1093/aje/kwq143)

Harley KG, Marks AR, Bradman A, Barr DB \& Eskenazi B 2008 DDT exposure, work in agriculture, and time-to-pregnancy among farmworkers in California. Journal of Occupational and Environmental Medicine 50 1335-1342. (doi:10.1097/JOM.0b013e31818f684d)

Harley KG, Marks AR, Chevrier J, Bradman A, Sjödin A \& Eskenazi B 2010 PBDE concentrations in women's serum and fecundability. Environmental Health Perspectives 118 699-704. (doi:10.1289/ehp. 0901450)

Ibáñez I, Potau N, Enriquez G \& de Zegher F 2000 Reduced uterine and ovarian size in adolescent girls born small for gestational age. Pediatric Research 47 575-577. (doi:10.1203/00006450-20000500000003)

Jensen TK, Jacobsen R, Christensen K, Nielsen NC \& Bostofte E 2009 Good semen quality and life expectancy: a cohort study of 43,277 men. American Journal of Epidemiology 170 559-565. (doi:10.1093/aje/ kwp168)

Jirsová S, Msaata J, Jech L \& Zvárová J 2010 Effect of polychlorinated biphenyls (PCBs) and 1,1,1-trichloro-2,2,-bis((4-chlorophenyl)-ethane
(DDT) in follicular fluid on the results of in vitro fertilization-embryo transfer (IVF-ET) programs. Fertility and Sterility 93 1831-1836. (doi:10.1016/j.fertnstert.2008.12.063)

Joffe M, Villard L, Li Z, Plowman R \& Vessey M 1993 Long-term recall of time-to-pregnancy. Fertility and Sterility 60 99-104.

Jönsson BA, Richthoff J, Rylander L, Giwercman A \& Hagmar L 2005 Urinary phthalates metabolites and biomarkers of reproductive function in young men. Epidemiology 16 487-493. (doi:10.1097/01.ede.0000 164555.19041.01)

Jørgensen N, Andersen AG, Eustache F, Irvine DS, Suominen J, Peterson JH, Andersen AN, Auger J, Cawood EH, Horte A et al. 2001 Regional differences in semen quality in Europe. Human Reproduction $\mathbf{1 6}$ 1012-1019. (doi:10.1093/humrep/16.5.1012)

Juul S, Karmaus W, Olsen J \& The European Infertility and Subfecundity Study Group 1999 Regional differences in waiting time to pregnancy: pregnancy-based surveys from Denmark, France, Germany, Italy and Sweden. Human Reproduction 14 1250-1254. (doi:10.1093/humrep/ 14.5.1250)

Lutz W, O'Neill BC \& Scherbov S 2003 Demographics. Europe's population at a turning point. Science 299 1991-1992. (doi:10.1126/science. 1080316)

Mendiola J, Jørgensen N, Andersson A-M, Calafat AM, Ye X, Redmon JB, Drobnis EZ, Wang C, Sparks A, Thurston SW et al. 2010 Are environmental levels of bisphenol A associated with reproductive function in fertile men? Environmental Health Perspectives 118 1286-1291. (doi:10.1289/ehp.1002037)

Mok-Lin E, Ehrlich S, Williams PL, Petrozza J, Wright DL, Calafat AM, Ye X \& Hauser R 2010 Urinary bisphenol A concentrations and ovarian response among women undergoing IVF. International Journal of Andrology 33 385-393. (doi:10.1111/j.1365-2605.2009.01014.x)

Pandolfi C, Zugaro A, Lattanzio F, Necozione S, Barbonetti A, Colangeli MS, Francavilla S \& Francavilla F 2008 Low birth weight and later development of insulin resistance and biochemical/clinical features of polycystic ovary syndrome. Metabolism 57 999-1004. (doi:10.1016/j.metabol.2008.02.018)

Priskorn L, Holmboe SA, Jacobsen R, Jensen TK, Lassen TH \& Skakkebaek NE 2012 Increasing trends in childlessness in recent birth cohorts - a registry-based study of the total Danish male population born from 1945 to 1980. International Journal of Andrology 35 449-455. (doi:10.1111/j.1365-2605.2012.01265.x)

Punab M, Zilaitiene B, Jørgensen N, Horte A, Matulevicius V, Peetsalu A \& Skakkebaek NE 2002 Regional differences in semen qualities in the Baltic region. International Journal of Andrology 25 243-252. (doi:10.1046/j.1365-2605.2002.00359.x)

Ridout MS \& Morgan BJ 1991 Modeling digit preferences in fecundability studies. Biometrics 47 1423-1433. (doi:10.2307/2532396)

Rothman KJ, Wise LA, Sørensen HT, Riis AH, Mikkelsen EM \& Hatch EE 2013 Volitional determinants and age-related decline in fecundability: a general population prospective cohort study in Denmark. Fertility and Sterility 99 1958-1964. (doi:10.1016/j.fertnstert.2013.02.040)

Sanin L-H, Carrasquilla G, Solomon KR, Cole DC \& Marshal EJ 2009 Regional differences in time to pregnancy among fertile women from five Colombian regions with different use of glyphosate. Journal of Toxicology and Environmental Health. Part A 72 949-960. (doi:10.1080/ 15287390902929691)

Saravelos SH, Cocksedge KA \& Li TC 2008 Prevalence and diagnosis of congenital uterine anomalies in women with reproductive failure: a critical appraisal. Human Reproduction Update 14 415-429. (doi:10.1093/humupd/dmn018)

Skakkebaek NE, Rajpert-DeMeyts E \& Main KM 2001 Testicular dysgenesis syndrome: an increasingly common developmental disorder with environmental aspects. Human Reproduction 16 972-978. (doi:10.1093/humrep/16.5.972)

Skakkebaek NE, Jørgensen N, Main KM, Rajpert-De Meyts E, Leffers $H$, Andersson AM, Juul A, Carlsen E, Mortensen GK, Jensen TK et al. 2006 Is human fecundity declining? International Journal of Andrology 29 2-11. (doi:10.1111/j.1365-2605.2005.00573.x)

Skakkebaek NE, Rajpert-De Meyts E, Jørgensen N, Main KM, Leffers $\mathbf{H}$, Andersson AM, Juul A, Jensen TK \& Toppari J 2007 Testicular cancer trends as 'whistel blowers' of testicular developmental problems in populations. International Journal of Andrology 30 198-204. (doi:10.1111/j.1365-2605.2007.00776.x) 
Swan SH, Brazil C, Drobinis EZ, Liu F, Kruse RL, Hatch M, Redmon JB, Wang C \& Overstreet JW 2003 Geographic differences in semen quality of fertile US males. Environmental Health Perspectives 111 414-420. (doi:10.1289/ehp.5927)

Talbott EO, Zborowski JV, Rager JR, Boudreaux MY, Edmundowicz DA \& Guzick DS 2004 Evidence for an association between metabolic cardiovascular syndrome and coronary and aortic calcification among women with polycystic ovary syndrome. Journal of Clinical Endocrinology and Metabolism 89 5454-5461. (doi:10.1210/jc.2003-032237)

Thoma ME, McLain AC, Louis JF, King RB, Trumble AC, Sundaram R \& Buck Louis GM 2013 The prevalence of infertility in the United States as estimated by the current duration approach and a traditional constructed approach. Fertility and Sterility 99 1324-1331. (doi:10.1016/j.fertnstert. 2012.11.037)

Tobias DK, Chavarro J, Williams MA, Buck Louis GM, Hu FB, RichEdwards J, Missmer S \& Zhang C 2013 History of infertility and risk of gestational diabetes mellitus: a prospective analysis of 40,773. American Journal of Epidemiology 178 1219-1225. (doi:10.1093/aje/kwt110)

Trussell JC \& Lee PA 2004 The relationship of cryptorchidism to fertility. Current Urology Reports 5 142-148. (doi:10.1007/s11934-004-0028-4)

Vestergaard S, Nielsen F, Andersson A-M, Hjollund NH, Grandjean P, Raun Andersen H \& Jensen TK 2012 Association between perfluorinated compounds and time to pregnancy in a prospective cohort of Danish couples attempting to conceive. Human Reproduction 27 873-880. (doi:10.1093/humrep/der450)
Wagner $\mathrm{U}$, Schlebusch $\mathrm{H}$, van der Ven $\mathrm{H}$, van der Ven $\mathrm{K}$, Diedrich $\mathrm{K}$ \& Krebs D 1990 Accumulation of pollutants in the genital tract of sterility patients. Journal of Clinical Chemistry and Clinical Biochemistry 28 683-688.

Whitworth KW, Haug LS, Baird DD, Becher G, Hoppin JA, Skjaerven R, Thomsen C, Eggesbo M, Travlos G, Wilson R et al. 2012 Perfluorinated compounds and subfecundity in pregnant women. Epidemiology 23 257-263. (doi:10.1097/EDE.0b013e31823b5031)

Zieluis GA, Hulscher ME \& Florack EI 1992 Validity and reliability of a questionnaire on fecundability. International Journal of Epidemiology 21 1151-1156. (doi:10.1093/ije/21.6.1151)

Zinman MJ, Clegg ED, Brown CC, O'Connor J \& Selevan SG 1996 Estimates of human fertility and pregnancy loss. Fertility and Sterility 65 503-509.

Zou Z, Hu H, Song M, Shen Y, Guo X, McElreavey K, Bittles AH \& Wang W 2011 Semen quality analysis of military personnel from six geographical areas of the People's Republic of China. Fertility and Sterility 95 2018-2023. (doi:10.1016/j.fertnstert.2011.02.052)

Received 23 September 2013

First decision 29 October 2013

Revised manuscript received 12 November 2013

Accepted 5 December 2013 